\title{
„Man kann der Patientin Mut machen, dass sie sich danach nicht mehr rasieren muss"
}

\author{
Mit der Photoepilation kann vielen Patientinnen mit stärkerer Behaarung dauerhaft geholfen \\ werden. Prof. Hans Wolff, Dermatologe an der LMU München, rät, das Haarwachstum zuvor \\ mit einer Wachsepilation zu synchronisieren; damit könne man die Effizienz deutlich steigern.
}

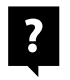

Prof. Dr. med. Hans Wolff Klinik und Poliklinik für Dermatologie und Allergologie, LMU München

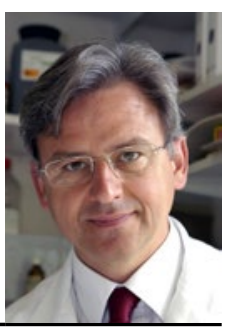

MMW: Was raten Sie Patientinnen mit Hirsutismus, die in die dermatologische Praxis kommen?

Wolff: Zuerst einmal kläre ich die Patientin über die einfachen symptomatischen Verfahren auf: Rasur, Bleichen der Haare, Wachsepilation, chemische Auflösung durch Thioglykolate usw. Eine Chance auf eine dauerhafte Haarentfernung hat man z.B. mit der Elektrolysebehandlung; diese funktioniert aber nur, wenn es sich um nicht allzu viele Haare handelt. Gerade beim Hirsutismus setzt sich mehr und mehr die Laser- oder Blitzlampenepilation durch.

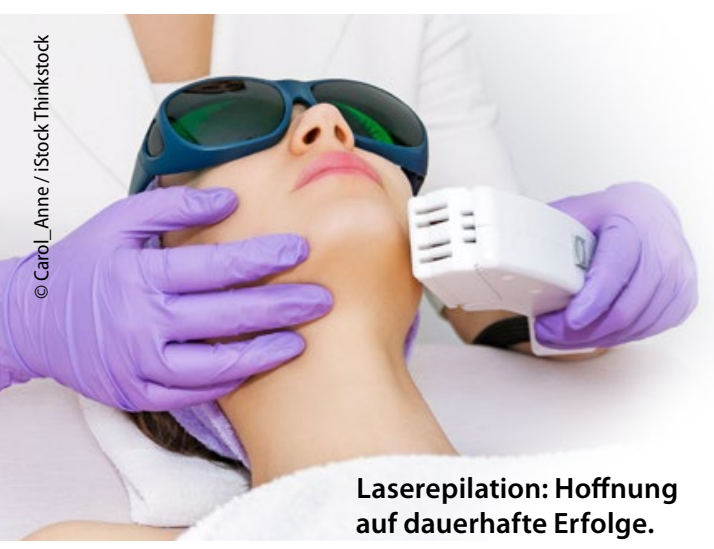

MMW: Was muss man beachten, wenn man einen Gesichtshirsutismus mit Photoepilation behandelt?

Wolff: In der Heilungsphase muss man unbedingt UV-Einflüsse vermeiden. Bei der Laserepilation kommt es im Zuge der Wundheilung zu Rötungen und manchmal sogar Krustenbildung. Werden diese Areale UV-Licht ausgesetzt, hat man ein hohes Risiko für Hyperpigmentierungen. Ich rate, eine Lasertherapie im Gesicht wenn möglich in die Wintermonate zu legen. Ein möglichst kompletter Sonnenschutz für zwei bis drei Monate ist das $\mathrm{A}$ und $\mathrm{O}$ !

\section{MMW: Der Erfolg des} Laserns hängt ja auch davon ab, wie hoch der Anteil der Haare ist, die sich in der anagenen Phase, also der für den Laser empfindlichen Wachstumsphase befinden. Wie geht man damit um?

Wolff: Man könnte die betroffenen Körperregionen durch eine Wachsepilation synchronisieren. Wenn man alle Haare ausreißt, werden vier bis acht Wochen später $100 \%$ der nachgewachsenen Haare im anagenen Stadium sein. Bei einer Laserepilation zu diesem Zeitpunkt hätte man eine viel höhere Effizienz und könnte die Zahl der Eingriffe minimieren das wäre auch kostensparend.

MMW: Wie dauerhaft ist die Wirkung der Laserepilation wirklich? In einer
Londoner Studie* von 2015 mussten sich die Patientinnen spätestens ein bis zwei Jahre nach der Laserepilation wieder regelmäßig enthaaren, und zwar fünfmal die Woche.

Wolff: Das halte ich heute für zu pessimistisch. In der DDG-Leitlinie von $2007^{\star *}$, an der ich beteiligt war, haben wir zwar auch noch einen sehr vorsichtigen Ton angeschlagen. Man kann aber heute wirklich vielen Frauen dauerhaft helfen, sofern man etwa sechs bis zehn Eingriffe vornimmt. Angesichts der technischen Weiterentwicklung kann man der Patientin ruhig Mut machen, dass sie sich danach nicht mehr ständig rasieren muss.

\section{vermeiden.}

\section{MMW: Schädigen die wiederholten Sitzungen} die Hautbeschaffenheit?

Wolff: Nein. Die Photothermolyse wirkt selektiv, das heißt, dass sie vor allem das konzentrierte Melanin im Haarfollikel anspricht. Zumindest bei hellhäutigen Frauen vom kaukasischen Typ befindet sich nur wenig Melanin in der Epidermis; daher bekommt diese so gut wie keine Laserenergie ab. Im Normalfall regeneriert sich die das Haar umgebende Haut komplett, sodass mit keinerlei Spätauswirkungen zu rechnen ist. -

Interview: Dr. Elke Oberhofer

* Roche A et al. Clin Experimental Dermatol 2015; doi: 10.1111/ced.12775

**Kunte C et al. Leitlinie der Deutschen Gesellschaft für Dermatologie: Therapy of Hypertrichosis. JDDG 2007;9:807-10 